\title{
On the Hausdorff dimension of certain self-affine sets
}

\author{
by
}

\author{
A. G. Abercrombie and R. Nair (Liverpool)
}

\begin{abstract}
A subset $E$ of $\mathbb{R}^{n}$ is called self-affine with respect to a collection $\left\{\phi_{1}, \ldots, \phi_{t}\right\}$ of affinities if $E$ is the union of the sets $\phi_{1}(E), \ldots, \phi_{t}(E)$. For $S \subset \mathbb{R}^{n}$ let $\Phi(S)=$ $\bigcup_{1 \leq j \leq t} \phi_{j}(S)$. If $\Phi(S) \subset S$ let $E_{\Phi}(S)$ denote $\bigcap_{k \geq 0} \Phi^{k}(S)$. For given $\Phi$ consisting of contracting "pseudo-dilations" (affinities which preserve the directions of the coordinate axes) and subject to further mild technical restrictions we show that there exist self-affine sets $E_{\Phi}(S)$ of each Hausdorff dimension between zero and a positive number depending on $\Phi$. We also investigate in detail the special class of cases in $\mathbb{R}^{2}$, where the images of a fixed square under some maps $\phi_{1}, \ldots, \phi_{t}$ are some vertical and some horizontal rectangles of sides 1 and 2. This investigation is made possible by an extension of the method of calculating Hausdorff dimension developed by P. Billingsley.
\end{abstract}

1. Introduction. A subset $E$ of $\mathbb{R}^{n}$ is called self-affine with respect to a collection $\Phi=\left\{\phi_{j}: j \in \Lambda\right\}$ of affine maps if $E$ is the union of the sets $\phi_{j}(E)$ over $j \in \Lambda$. For any set $S \subset \mathbb{R}^{n}$ let $\Phi(S)$ denote $\bigcup_{j \in \Lambda} \phi_{j}(S)$ and if $\Phi(S) \subset S$ let $E_{\Phi}(S)$ denote $\bigcap_{k>0} \Phi^{k}(S)$. It is straightforward to check that $E_{\Phi}(S)$ is self-affine with respect to $\Phi$. A number of authors have considered, in varying degrees of generality, the problem of determining or estimating the Hausdorff dimension of self-affine sets of the type $E_{\Phi}(S)$, where the index set $\Lambda$ is finite, $S$ is compact and the sets $\phi_{j}(S)$ are quasi-disjoint, that is, we have

$$
\operatorname{int}\left(\phi_{i}(S)\right) \cap \operatorname{int}\left(\phi_{j}(S)\right)=\emptyset \quad \text { when } i \neq j .
$$

(See Section 2 for a definition of Hausdorff dimension.) For generic selfaffine sets the answer was obtained by K. Falconer [Fa1], but for specific self-affine sets the problem of determining the exact Hausdorff dimension of $E_{\Phi}(S)$ is apparently still hard and in only a few cases are results known. For instance the case where all the $\phi_{j}$ are similarities is treated in [Be] and the case where for each $i, j$ there is a translation $\psi_{i, j}$ such that $\phi_{i}=\psi_{i, j} \phi_{j}$ is treated in [Bed]. Bounds for the Hausdorff dimension are also available however [Fa2]. See also the work of C. McMullen [McM]. For the case $n=2$,

2000 Mathematics Subject Classification: Primary 28A75. 
I. Hueter and S. P. Lally [HL] give some rather general sufficient conditions for the upper bound in [Fa2] to be exact.

In this paper we consider in greater detail the situation where $\Phi$ consists only of pseudo-dilations, that is, affinities which preserve the directions of the coordinate axes in $\mathbb{R}^{n}$, but we shall not restrict ourselves to finite $\Lambda$ or compact sets $S$. We will address the problem of determining the range of values taken by the Hausdorff dimension $\operatorname{dim} E_{\Phi}(S)$ as $S$ ranges over the bounded subsets of $\mathbb{R}^{n}$. Theorem 1.2 represents a partial solution to this problem, and shows that under certain conditions we can find bounded sets self-affine with respect to $\Phi$ and having arbitrary Hausdorff dimension between zero and a positive number depending on $\Phi$.

We note in passing that, since the conditions in [HL] include the condition that for some angular sector $A$ of $\mathbb{R}^{2}$ the inverse images $\phi_{j}^{-1}(A)$ should be pairwise disjoint subsets of $A$, the results of that paper have little bearing in our context even for compact $S$. As is normal with problems of this type most of the work goes into obtaining lower bounds, while elementary covering arguments suffice for the upper bound.

Let $Q$ be the unit cube in $\mathbb{R}^{n}$, so $Q=[0,1]^{n}$. Let $\Phi$ be a set of pseudodilations of $\mathbb{R}^{n}$ such that the images $\phi(Q)$, for $\phi$ in $\Phi$, are pairwise quasidisjoint subsets of $Q$. Then $\Phi$ is necessarily (at most) countable so with no loss of generality we can assume $\Phi$ is a sequence $\left(\phi_{j}\right)_{j \in \Lambda}$ where $\Lambda$ is either $\{1, \ldots, k\}$ for some positive integer $k$ or possibly $\Lambda=\mathbb{N}$. For $S \subset \mathbb{R}^{n}$ we set $\Phi(S)=\bigcup_{j \in \Lambda} \phi_{j}(S)$ and set $E_{\Phi}(S)=\bigcap_{n \in \mathbb{N}} \Phi^{n}(S)$.

For a linear map $\phi$ from $\mathbb{R}^{n}$ to itself which we assume to be contracting and non-singular, we refer to its singular values $\alpha_{i}(1 \leq i \leq n)$ as the lengths of the mutually perpendicular principal semi-axes of $\phi(B)$, where $B$ is the unit ball in $\mathbb{R}^{n}$. We adopt the convention that $\alpha_{1} \geq \ldots \geq \alpha_{n}$. Since $\phi$ is contracting and non-singular we have $\alpha_{1}<1$ and $\alpha_{n}>0$. For $0 \leq s \leq n$ we define the singular value function of $\phi$ to be

$$
L^{s}(\phi)=\alpha_{1} \ldots \alpha_{m-1} \alpha_{m}^{s-m+1},
$$

where $m$ is the integer such that $m-1<s \leq m$. We also assign the value

$$
L^{s}(\phi)=\alpha_{1} \ldots \alpha_{n}=(\operatorname{det} \phi)^{s / n}
$$

if $s>n$. It is clear that $L^{s}(\phi)$ is continuous and strictly decreasing in $s$. If $s$ is an integer and $0 \leq s \leq n$ then

$$
L^{s}(\phi)=\alpha_{1} \ldots \alpha_{s}=\sup _{E} \frac{m_{s}(\phi(E))}{m_{s}(E)},
$$

where $m_{s}$ denotes $s$-dimensional Lebesgue measure and the supremum is taken over all possible $s$-dimensional ellipsoids $E$ in $\mathbb{R}^{n}$. For $r=0,1,2, \ldots$ let

$$
J_{r}=\left\{\left(j_{1}, \ldots, j_{r}\right): j_{i} \in \Lambda\right\}
$$


and let $d(\Phi)$ denote the unique real number $s$ such that

$$
\lim _{r \rightarrow \infty}\left(\sum_{\left(j_{1}, \ldots, j_{r}\right) \in J_{r}} L^{s}\left(\phi_{i_{1}}, \ldots, \phi_{i_{r}}\right)\right)^{1 / r}=1 .
$$

We note that if the $\phi_{j}$ are pseudo-dilations each $\phi_{j}$ can be written uniquely as $\sigma_{j} \circ \psi_{j}$ where $\sigma_{j}$ is a translation and $\psi_{j}$ is given by the matrix $\operatorname{diag}\left(a_{i}^{(j)}\right)_{1 \leq i \leq n}$. Let $\mathcal{T}$ denote the set of probability vectors in $[0,1]^{\Lambda}$; that is, the elements of $\mathcal{T}$ are sequences $T=\left(\tau_{j}\right)_{j \in \Lambda}$ with $\sum_{j \in \Lambda} \tau_{j}=1$. For $T$ in $\mathcal{T}$ we write

$$
h(T)=\sum_{j \in \Lambda} \tau_{j}\left|\log \tau_{j}\right|
$$

and for $1 \leq i \leq n$ we write

$$
a_{i}(T)=\sum_{j \in \Lambda} a_{i}^{(j)} \tau_{j}
$$

We define $\mathcal{T}_{\Phi}$ to be the set

$$
\left\{T \in \mathcal{T}: h(T)<\infty, a_{i}(T)<\infty \text { for } 1 \leq i \leq n\right\} .
$$

For $T \in \mathcal{T}_{\Phi}$ we set

$$
f(T)=f_{\Phi}(T)=n+\frac{h(T)-\sum_{1 \leq i \leq n} a_{i}(T)}{\min _{1 \leq i \leq n} a_{i}(T)} .
$$

We have the following theorem.

Theorem 1.1. We have

$$
\sup _{T \in \mathcal{T}_{\Phi}} f(T) \leq \operatorname{dim} E_{\Phi}(Q) \leq \min \{n, d(\Phi)\} .
$$

Fix $\Phi=\left(\phi_{j}\right)_{j \in \Lambda}$ as above and let $S$ be any bounded subset of $\mathbb{R}^{n}$ that is self-affine with respect to $\Phi$. Is it necessarily the case that $\operatorname{dim} S \leq$ $\operatorname{dim} E_{\Phi}(Q)$ ? If $\Lambda$ is finite, we have $\max _{i, j} a_{i}^{(j)}<1$ and it follows easily that $S \subset Q$ and so by self-affinity $S \subset E_{\Phi}(Q)$ and therefore $\operatorname{dim} S \leq \operatorname{dim} E_{\Phi}(Q)$ in this case. However if $\Lambda=\mathbb{N}$ we may have $\sup _{i, j} a_{i}^{(j)}=1$ and then it is possible that $S$ is not contained in $Q$. Moreover although $S$ is certainly contained in some cube $Q^{\prime}$ such that $\Phi\left(Q^{\prime}\right)$ is a subset of $Q^{\prime}$, the images $\phi_{i}\left(Q^{\prime}\right)$ may not be quasi-disjoint, so we cannot appeal to a change of scale to assure ourselves that $\operatorname{dim} S \leq \operatorname{dim} E_{\Phi}(Q)$ in the case $\Lambda=\mathbb{N}$, and it remains an open question whether possibly $\operatorname{dim} S>\operatorname{dim} E_{\Phi}(Q)$ in the case of infinite $\Lambda$.

It seems natural to enquire whether the Hausdorff dimension of $S$ can take any value between zero and the Hausdorff dimension of $E_{\Phi}(Q)$. In this paper we prove a theorem which gives a partial affirmative answer to this question. In the context of the following theorem we assume that $\Phi$ satisfies the following hypothesis: 
(C) there exists $T$ in $\mathcal{T}$ such that

$$
a_{1}(T)=\ldots=a_{n}(T) .
$$

This condition may seem rather restrictive. However for $|\Lambda| \geq n$, if a set of $|\Lambda|$ contracting affine maps given by diagonal matrices is chosen "at random" (with respect to Lebesgue measure on the space of coefficients) it is easily checked that condition (C) holds with positive probability.

For $\Phi$ satisfying $(\mathrm{C})$ set

$$
\mathcal{T}_{\Phi}^{*}=\left\{T \in \mathcal{T}: a_{1}(T)=\ldots=a_{n}(T)\right\},
$$

and choose $T_{0}$ in $\mathcal{T}_{\Phi}^{*}$ such that

$$
f\left(T_{0}\right)=\max _{T \in \mathcal{T}_{\phi}^{*}} f(T),
$$

as is possible as a consequence of the compactness of $[0,1]^{\Lambda}$ and the continuity of $f$.

TheOREM 1.2. Suppose $\Phi$ satisfies hypothesis (C) above, that $T_{0}$ is as just defined and $f$ is as in Theorem 1.1. Then for each $d$ in $\left[0, f\left(T_{0}\right)\right]$ there exists a set $S_{d}$ which is self-affine with respect to $\Phi$ such that the Hausdorff dimension of $S_{d}$ is $d$.

In order to shed some light on the nature of these theorems, the general cases of which are at present computationally intractable, we will investigate in some detail the following special class of examples. Let $n=2$, fix $a<1 / 2$, and suppose that for each $j$ in $\Lambda$ the image $\phi_{j}(Q)$ is either a vertical or horizontal rectangle of sides $a$ and $2 a$ (a "domino"). Clearly $\Lambda$ must be finite. If all the rectangles $\phi_{j}(Q)$ are vertical or all are horizontal we are in the situation of [Bed] so we direct our attention to the problem of finding bounds for $\operatorname{dim} E_{\Phi}(Q)$ when there is at least one rectangle of each kind. This will reveal some of the strengths and limitations of Theorems 1.1 and 1.2 and will suggest further problems.

It is possible that the methods of this paper could be used to yield non-trivial results for more general classes of self-affine sets than those we consider, but there remain considerable obstacles to overcome, arising notably from the fact that whereas pseudo-dilations with a common fixed point form an abelian group, affine maps with a common fixed point do not.

In two papers [Bi1], [Bi2] dating from the early 1960's, P. Billingsley developed a concept of dimension in probability spaces akin to that of Hausdorff dimension in metric space. With the aid of a powerful comparison principle (Theorem 2.2 of [Bi2]) he obtained simple proofs of some classical results on the Hausdorff dimension of certain exceptional sets as well as a number of new results in the same spirit. The natural setting for the concept 
of Billingsley dimension seems to be in the context of zero-dimensional topological spaces. See for instance [Bi1], [Bi2], [Caj], [Ab1], [Ab2], [Ab3]. While ad hoc techniques exist ([Bi3], [KP], [AN1]) whereby results on Hausdorff dimension can be read off in $[0,1]$ from results on Billingsley dimension in an appropriate sequence space, these techniques do not seem to transfer to problems in $[0,1]^{d}$ for $d>1$ where the geometry interferes more. The results of this paper arise by adapting the method developed in [AN2] to overcome this difficulty in the context of expanding maps of manifolds.

The plan of the paper is that in $\S 2$ we describe the notions of Hausdorff dimension and Billingsley dimension, in $\S 3$ we describe our generalisation of Billingsley dimension and we state a general comparison principle, in $\S 4$ we prove Theorem 1.1, in $\S 5$ we prove Theorem 1.2 and in $\S 6$ we study the special class of examples mentioned above.

2. Billingsley dimension in terms of zero-dimensional nets. For any subset $A$ of $\mathbb{R}$ let $|A|$ denote its diameter, that is,

$$
|A|=\sup \{|x-y|: x, y \in A\} .
$$

Let $E$ be any subset of $\mathbb{R}$ and $t$ a positive number. For each positive $\delta$, we consider the possible countable coverings $\left\{A_{j}\right\}$ of $E$ by sets of diameter less than $\delta$. We define

$$
m_{t}^{\delta}(E)=\inf \left\{\sum_{j}\left|A_{j}\right|^{t}:\left|A_{j}\right|<\delta, E \subset \bigcup_{j} A_{j}\right\} .
$$

Here the infimum is taken over all possible such covers of $E$. As $\delta$ decreases, the class of such coverings of $E$ diminishes, so $m_{t}^{\delta}(E)$ increases and we set

$$
m_{t}(E)=\lim _{\delta \rightarrow 0} m_{t}^{\delta}(E)=\sup _{\delta>0} m_{t}^{\delta}(E) .
$$

The limit always exists (we allow it to take the value $\infty$ ), and $m_{t}(E)$ is called the $t$-dimensional Hausdorff measure of $E$. Technically $m_{t}$ is in fact an outer measure on $\mathbb{R}$, which defines a Borel measure. As the reader will readily verify, if $m_{t}(E)<\infty$ and $t<T$, then $m_{T}(E)=0$. As an immediate consequence we see that there exists a non-negative number $\operatorname{dim} E$ such that

$$
m_{t}(E)= \begin{cases}\infty & \text { if } t<\operatorname{dim} E, \\ 0 & \text { if } t>\operatorname{dim} E,\end{cases}
$$

and we call $\operatorname{dim} E$ the Hausdorff dimension of $E$. (The value $m_{t}(E)$ takes may lie anywhere in $[0, \infty)$.) Hausdorff dimension is interesting as a refinement of our measure of size because if a subset of $\mathbb{R}$ has positive Lebesgue measure then it has Hausdorff dimension one, while there exist sets of zero Lebesgue measure and Hausdorff dimension $t$ for any $t$ in $[0,1]$. This is not the only possible definition of dimension. However it is probably the geometric definition best underpinned by rigorous foundations and also the 
best known and so we confine attention to it. This said, for our purposes, as a technical device, it is convenient to work with the related concept of Billingsley dimension which we now define.

Let $X$ be a compact Hausdorff space. We define a zero-dimensional net over $X$ to be a collection $\mathcal{P}$ of regular closed non-empty subsets of $X$ with the following properties:

(N1) $\quad X \in \mathcal{P}$;

(N2) if $\mathcal{C} \subset \mathcal{P}$ is a chain of subsets of $X$ ordered by inclusion then $\bigcup_{P \in \mathcal{C}} P$ is in $\mathcal{C}$;

$\left(\mathrm{N} 3_{0}\right) \quad$ if $u$ and $v$ are elements of $\mathcal{P}$ then $u \subset v$ or $v \subset u$ or $u \cap v=\emptyset$;

(N4) if $\mathcal{C}$ is a maximal chain (ordered by inclusion) in $\mathcal{P}, x \in \bigcap_{P \in \mathcal{C}} P$ and $U$ is a neighbourhood of $x$, then for some $P \in \mathcal{C}$ we have $P \subset U$.

The reason for calling such a structure "zero-dimensional" is as follows. Let $X_{\mathcal{P}}$ be the set of $x$ in $X$ that are in $\bigcap_{P \in \mathcal{C}} P$ for some maximal $\mathcal{C}$ in $\mathcal{P}$. We readily see that $X_{\mathcal{P}}$ is a zero-dimensional subset of $X$. For example if $X$ is the interval $[0,1]$ with the usual topology we can take $\mathcal{P}$ to be the collection of intervals

$[0,1], \quad[0,1 / 3], \quad[2 / 3,1], \quad[0,1 / 9], \quad[2 / 9,1 / 3], \quad[2 / 3,7 / 9], \quad[8 / 9,1], \ldots$

Then $\mathcal{P}$ forms a zero-dimensional net and $X_{\mathcal{P}}$ is the Cantor set. Again if $X$ is $[0,1)$ with the "right open topology" [SS, p. 75$]$ we can take $\mathcal{P}$ to be the collection of dyadic intervals

$$
[0,1), \quad[0,1 / 2), \quad[1 / 2,1), \quad[0,1 / 4), \ldots,
$$

and we have $X_{\mathcal{P}}=X$. Let $\mu$ be any non-atomic Borel probability measure on $X_{\mathcal{P}}$ and $E$ any subset of $X_{\mathcal{P}}$. Set

$$
\ell_{\mu, \theta}^{\gamma}(E)=\inf _{\mathcal{C}} \sum_{B \in \mathcal{C}}(\mu(B))^{\gamma}
$$

where the infimum is taken over all covers $\mathcal{C}$ of $E$ in $\mathcal{P}$ satisfying $\mu(B) \leq \theta$ for each $B \in \mathcal{C}$. Again we can write

$$
\ell_{\mu}^{\gamma}(E)=\lim _{\theta \rightarrow 0} \ell_{\mu, \theta}^{\gamma}(E)=\sup _{\theta>0} \ell_{\mu, \theta}^{\gamma}(E) .
$$

As before ([Bi3], pp. 136-137), there exists a non-negative real number $\operatorname{dim}_{\mu}^{\mathcal{P}}(E)$ such that

$$
\ell_{\mu}^{\gamma}(E)= \begin{cases}\infty & \text { if } \gamma<\operatorname{dim}_{\mu}^{\mathcal{P}}(E), \\ 0 & \text { if } \gamma>\operatorname{dim}_{\mu}^{\mathcal{P}}(E) .\end{cases}
$$

We call $\operatorname{dim}_{\lambda}^{\mathcal{P}}(E)$ the Billingsley dimension of $E$ with respect to $\mathcal{P}$ and $\mu$. For certain choices of $\mathcal{P}$ the Billingsley dimension of a set with respect to $\mathcal{P}$ and Lebesgue measure $\lambda$ coincides with its Hausdorff dimension. Because of (N2), 
every chain $\mathcal{C}$ contained in $\mathcal{P}$ is well-ordered by $\supset$ and so there is a canonical isomorphism $i_{\mathcal{C}}$ (say) for some ordinal $\alpha=\alpha(\mathcal{C})$ to the chain $\mathcal{C}$ ordered by $\supset$. If $x \in X_{\mathcal{P}}$ denote the set $\{P \in \mathcal{P}: x \in P\}$ by $\mathcal{P}_{x}$. Because of $\left(\mathrm{N} 3_{0}\right)$ the set $\mathcal{P}_{x}$ is a chain for each $x$ in $X$. For any $\beta$ in $\alpha\left(\mathcal{P}_{x}\right)$ write $P_{\beta}(x)=i_{\mathcal{P}_{x}}(\beta)$. A specialised form of the following theorem is given in [Bi1] and [Bi2]. The proof given there carries over to the present setting. We make the convention that for $a$ and $b$ in $[0,1]$ if either $a$ or $b$ is in $\{0,1\}$ we set $\frac{\log a}{\log b}=1,0$ or $\infty$ according as $a>b, a=b$, or $a<b$. We have the following theorem due to Billingsley.

THEOREM 2.1. Let $\mu, \nu$ be non-atomic Borel probability measures on a compact Hausdorff space $X$ and let $\mathcal{P}$ be a countable net over $X$. Suppose $E \subset X_{\mathcal{P}}$ and $\delta \geq 0$ satisfies

$$
\liminf _{\beta \rightarrow \alpha\left(\mathcal{P}_{x}\right)} \frac{\log \nu\left(P_{\beta}(x)\right)}{\log \mu\left(P_{\beta}(x)\right)} \geq \delta \quad \text { for all } x \text { in } E .
$$

Then $\operatorname{dim}_{\mu}^{\mathcal{P}}(E) \geq \delta \operatorname{dim}_{\nu}^{\mathcal{P}}(E)$.

The following variant of Theorem 2.1 is occasionally useful and will in particular be used in the proof of Theorem 1.2. It is obtained by two applications of Theorem 2.1 in one of which the roles of $\mu$ and $\nu$ are reversed.

Theorem 2.2. Let $\mu, \nu$ and $\mathcal{P}$ be as in Theorem 2.1. Suppose $E \subset X_{\mathcal{P}}$ and $\delta \geq 0$ satisfies

$$
\liminf _{\beta \rightarrow \alpha\left(\mathcal{P}_{\beta}(x)\right)} \frac{\log \nu\left(P_{\beta}(x)\right)}{\log \mu\left(P_{\beta}(x)\right)}=\delta \quad \text { for each } x \text { in } E .
$$

Then $\operatorname{dim}_{\mu}^{\mathcal{P}}(E)=\delta \operatorname{dim}_{\nu}^{\mathcal{P}}(E)$.

In applications of Theorem 2.1 we are usually seeking a lower bound for $\operatorname{dim}_{\mu}^{\mathcal{P}}(E)$ where $\mu$ is some naturally occurring measure (for instance $X$ may be a topological group with Haar measure $\mu$ ). One therefore aims to construct $\nu$ in such a way that both $\operatorname{dim}_{\nu}^{\mathcal{P}}(E)$ and the left hand side of $(2.2)$ are easy to compute.

3. Higher dimensional nets and a comparison principle. Let $X$ be a compact Hausdorff space. We call a family $\mathcal{P}$ of non-empty, regular closed subsets of $X$ a net if $\mathcal{P}$ satisfies (N1), (N2) and (N4) as well as (N3) for $P, Q \in \mathcal{P}$ we have $P \cap Q \in\{P, Q, \partial P \cap \partial Q\}$.

As before the set of $x \in X$ such that $x$ is in the intersection of some maximal chain $\mathcal{C} \subset \mathcal{P}$ is denoted by $X_{\mathcal{P}}$. The number $\max _{x \in X} \#\{P \in \mathcal{P}$ : $x \in \partial P\}$ may plausibly be called the dimension of $\mathcal{P}$. It is easy to check that a zero-dimensional net as defined in $\S 2$ is just a net of dimension zero. Henceforth $\mathcal{P}$ will always denote a net over $X$. For $P \in \mathcal{P}$, let $\widetilde{P}$ denote the set of maximal chains in $\mathcal{P}$ that contain $P$. Thus in particular $\widetilde{X}$ denotes 
the set of maximal chains in $\mathcal{P}$. We construct a zero-dimensional topology on $\widetilde{X}$. In $[$ AN2] we show that for $P, Q$ in $\mathcal{P}$, if $P \subset Q$ then $\widetilde{P} \subset \widetilde{Q}$. We write $\widetilde{\mathcal{P}}=\{\widetilde{P}: P \in \mathcal{P}\}$ and take $\widetilde{\mathcal{P}}$ as a subbasis for the topology on $\widetilde{X}$. In [AN2] it is shown that the set $\widetilde{\mathcal{P}}$ is a zero-dimensional net over $\widetilde{X}$.

By (N4) and the fact that $X$ is Hausdorff we have $\#\left(\bigcap_{P \in \varrho} P\right) \leq 1$ for each $\varrho$ in $\widetilde{X}$. On the other hand the fact that each $P$ in $\varrho$ is compact implies that $\#\left(\bigcap_{P \in \varrho} P\right) \geq 1$. Thus there is a surjection $\pi: \widetilde{X} \rightarrow X_{\mathcal{P}}$ given by $\pi(P)=\bigcap_{P \in \varrho} P$. Clearly we have $\pi(\widetilde{P})=P$ for each $P \in \mathcal{P}$ but not $\pi^{-1}(P)=\widetilde{P}$ in general.

For each $E \subset X_{\mathcal{P}}$ and a cover $\mathcal{Q} \subset \mathcal{P}$ of $E$ we say $\mathcal{Q}$ is saturated if for each $x \in E$, and each $\varrho$ in $\pi^{-1}(x), \mathcal{Q}$ contains a representative of $\varrho$. Suppose now that $\mu$ is a non-atomic probability measure on $X$. To define the $\operatorname{dimension} \operatorname{dim}_{\mu}^{\mathcal{P}}(E)$ of $E$ we write, for each $\gamma$ in $[0,1]$ and $\theta>0$,

$$
\ell_{\theta}^{\gamma}(E)=\inf _{\mathcal{Q}} \sum_{P \in \mathcal{Q}}(\mu(P))^{\gamma}
$$

where now the infimum is taken over the saturated covers $\mathcal{Q}$ contained in $\mathcal{P}$ of $E$ such that $\mu(P) \leq \theta$ for each $P \in \mathcal{Q}$. As before we write

$$
\ell^{\gamma}(E)=\lim _{\theta \rightarrow \infty} \ell_{\theta}^{\gamma}(E)
$$

and

$$
\operatorname{dim}_{\mu}^{\mathcal{P}}(E)=\sup \left\{\gamma: \ell^{\gamma}(E)=\infty\right\}=\inf \left\{\gamma: \ell^{\gamma}(E)=0\right\} .
$$

If $\mathcal{P}$ is zero-dimensional then every cover $\mathcal{C} \subset \mathcal{P}$ is saturated so our definition coincides with Billingsley's in this case. For an arbitrary net $\mathcal{P}$ we write $\partial \mathcal{P}=\bigcup_{P \in \mathcal{P}} \partial P$. The following is easily verified.

THEOREM 3.1. Let $\mu$ be a non-atomic Borel probability measure on a space $X$ and let $\mathcal{P}$ be a net over $X$ satisfying

$$
\mu(\partial \mathcal{P})=0 .
$$

Then $\mu \circ \pi$ is a non-atomic Borel probability measure on $\widetilde{X}$ and

$$
\operatorname{dim}_{\mu \circ \pi}^{\widetilde{\mathcal{P}}}(\Omega) \leq \operatorname{dim}_{\mu}^{\mathcal{P}}(\pi(\Omega)) \quad \text { for each } \Omega \subset \widetilde{X} .
$$

We now turn to the statement of a comparison principle. Let $(X, d)$ be a metric space. To avoid trivialities we wish to ensure that the set over which the infimum in (2.1) is taken is non-empty, that is, for some $\delta>0$, the set $X$ can be covered by countably many sets of diameter $\leq \delta$. It is convenient to assume that $X$ is compact. One consequence of this is that $X$ then contains at most finitely many isolated points, $x_{1}, \ldots, x_{r}$ say. It follows that $X^{\prime}=X \backslash\left\{x_{1}, \ldots, x_{r}\right\}$ is compact and has no isolated points. Such a space is called perfect. Since a finite set of points can have no bearing on questions 
of Hausdorff dimension we henceforth make the simplifying assumption that $X$ is itself perfect. The following is proved in [AN2]:

TheOREM 3.2. Let $\eta$ be a Borel probability measure on a perfect metric space $X$ satisfying

$$
\eta(E) \ll|E|^{\operatorname{dim}(E)}
$$

for all measurable $E$ contained in $X$. Let $\mathcal{P}$ be a net over $X$ satisfying $\left(\mathrm{N} 5_{\eta}\right)$ and also

(N6) for some $K>0$ and for each $P \in \mathcal{P}, P \neq X$, there exists $Q \in \mathcal{P}$ such that $P$ is strictly contained in $Q$ and $\eta(P) \geq K \eta(Q)$; and also for some $\alpha$ in $(\operatorname{dim} X, \infty)$,

$$
|P| \ll(\eta(P))^{1 / \alpha} \quad \text { for each } P \text { in } \mathcal{P} \text {. }
$$

Then for any $E \subset X_{\mathcal{P}}$ we have

$$
\operatorname{dim} E \geq \operatorname{dim} X-\alpha+\alpha \operatorname{dim}_{\eta}^{\mathcal{P}}(E) .
$$

4. Proof of Theorem 1.1. The upper bound can be proved using exactly the same method as that described in [Fa1] and so we make no further remark about it. To prove the lower bound we begin by observing that $\sup _{T \in \mathcal{T}_{\phi}} f(T)=\sup _{T \in \mathcal{T}_{0}} f(T)$ where we write $\mathcal{T}_{0}$ to denote $\{T \in \mathcal{T}$ : $\#(\operatorname{supp}(T))<\infty\}$. Here $\operatorname{supp}(T)$ means $\left\{j \in \Lambda: \tau_{j}>0\right\}$. To see this we argue as follows: for any $T \in \mathcal{T}_{0}$ and $m \geq \inf (\operatorname{supp}(T))$ we set $s_{m}=$ $\sum_{1<h<m} \tau_{h}$ and let $T_{m}$ denote the vector $\left(\chi_{[1, m]}(j) \tau_{j} / s_{j}\right)_{j \in \Lambda}$. Then $T_{m} \in \mathcal{T}_{0}$ for each $m$ and if $T_{m} \in \mathcal{T}_{\Phi}$ then it is easily verified that $h\left(T_{m}\right)$ tends to $h(T)$ and $a_{i}\left(T_{m}\right)$ tends to $a_{i}(T)$ for each $i$ in $[1, n]$, so that $f\left(T_{m}\right)$ tends to $f(T)$ and it follows as claimed that

$$
\sup _{T \in \mathcal{T}_{\phi}} f(T)=\sup _{T \in \mathcal{T}_{0}} f(T) .
$$

It thus remains to prove that $\operatorname{dim} E_{\Phi}(Q) \geq f(T)$ for each $T$ in $\mathcal{T}_{0}$.

Now for $T=\left(\tau_{j}\right)_{j \in \Lambda}$ in $\mathcal{T}_{0}$, let $\Phi_{1}$ denote $\left(\phi_{j}\right)_{j \in \operatorname{supp}(T)}$, so $\Phi_{1}$ is finite. Since $E_{\Phi_{1}}(Q) \subset E_{\Phi}(Q)$ we have only to show that $\operatorname{dim} E_{\Phi_{1}}(T) \geq f_{\Phi}(T)$. But we have $f_{\Phi}(T)=f_{\Phi_{1}}(T)$ and so the theorem will be proved completely as soon as it is proved for any finite $\Phi$. We therefore henceforth assume that $\Phi$ is finite, say $\Phi=\left(\phi_{j}\right)_{j=1}^{t}$.

In order to apply Theorems 3.1, 3.2 and the Billingsley comparison principle we make use of the net $\mathcal{P}$ as follows. Write $\mathcal{P}_{0}=Q$, and for $k>0$ write

$$
\mathcal{P}_{k}=\left\{\phi_{j}(P): P \in \mathcal{P}_{k-1}, 1 \leq j \leq t\right\} .
$$

We set $\mathcal{P}=\bigcup_{k>0} \mathcal{P}_{k}$. Observe that each $x$ in $E=E_{\phi}$ satisfies

$$
\{x\}=\bigcap_{k \geq 1} \phi_{i_{1}} \circ \ldots \circ \phi_{i_{k}}(Q)
$$


for some sequence $\left(i_{k}\right)$ in $t^{\mathbb{N}}$. We denote the set of sequences $\left(i_{k}\right)$ for which (4.1) holds by $W(x)$. Observe also that each $\varrho$ in $\widetilde{Q}$ has the form

$$
\left\{\phi_{i_{1}} \circ \ldots \circ \phi_{i_{k}}(Q): k \in \mathbb{N}\right\}
$$

for some $w(\varrho)=\left(i_{k}\right)$ in $t^{\mathbb{N}}$. Then $w(\varrho)$ being in $W(x)$ implies $\pi(\varrho)=x$. We denote by $\widetilde{P}\left(i_{1}, \ldots, i_{k}\right)$ the set of $\varrho$ in $\widetilde{Q}$ such that the first $k$ terms of $w(\varrho)$ are $i_{1}, \ldots, i_{k}$. Every element of $\widetilde{P}$ is of this form (we make the convention $\widetilde{Q}=\widetilde{T}(\phi))$ and we have $\pi\left(\widetilde{P}\left(i_{1}, \ldots, i_{k}\right)\right)=\phi_{i_{1}} \circ \ldots \circ \phi_{i_{k}}(Q)$. Now for $T$ in $\mathcal{T}$ and $1 \leq h \leq t$ define

$$
A_{h}(T)=\left\{\left(i_{k}\right)_{k=1}^{\infty}:\left|\#\left(\left\{j \leq k: i_{j}=h\right\}\right)-k \tau_{h}\right| \ll(k \log k)^{1 / 2}\right\}
$$

and set

$$
\begin{gathered}
E_{h}(T)=\left\{x \in E: W(x) \cap A_{h}(T) \neq \emptyset\right\}, \\
\widetilde{Q}_{h}(T)=\left\{\varrho \in \widetilde{Q}: W(\varrho) \in A_{h}(T)\right\}, \\
A(T)=\bigcap_{1 \leq h \leq t} A_{h}(T), \quad E(T)=\bigcap_{1 \leq h \leq t} E_{h}(T), \quad \widetilde{Q}(T)=\bigcap_{1 \leq h \leq t} \widetilde{Q}_{h}(T) .
\end{gathered}
$$

To find the lower bound for the Hausdorff dimension of $E(T)$ we shall estimate

$$
\operatorname{dim}_{\lambda \circ \pi}^{\widetilde{\mathcal{P}}}(\widetilde{E}(T)),
$$

using the comparison principle, where $\lambda$ denotes $n$-dimensional Lebesgue measure. Define $\nu: \widetilde{\mathcal{P}} \rightarrow[0,1]$ recursively by setting $\nu(\widetilde{Q})=1$ and

$$
\nu\left(\widetilde{P}\left(i_{1}, \ldots i_{k}\right)\right)=\tau_{i_{k}} \nu\left(\widetilde{P}\left(i_{1}, \ldots i_{k-1}\right)\right)
$$

for $k>1$. One checks that $\nu$ is finitely additive and so, by a special case ([Pa], p. 139) of Kolmogorov's consistency theorem, can be extended to a Borel probability measure (also denoted by $\nu$ ) on $\widetilde{Q}$. Provided the cardinality of the support of $T$ is greater than one (the statement that $\operatorname{dim} E_{\Phi}(Q) \geq f(T)$ is easily seen to be trivial otherwise), the measure $\nu$ is also non-atomic. Now $\nu\left(\widetilde{Q}_{h}(T)\right)$ is just the probability that in the sequence of Bernoulli trials with probability of success $\tau_{h}$ the number of successes in the first $k$ trials is $\left.k \tau_{h}+O(k \log k)^{1 / 2}\right)$ as $k \rightarrow \infty$. By elementary probability theory ([Fel], p. 203) we therefore have $\nu\left(\widetilde{Q}_{h}(T)\right)=1$ for each $h$ and consequently $\nu(\widetilde{Q}(T))=1$ also. This implies $\operatorname{dim}_{\nu}^{\mathcal{P}}(\widetilde{Q}(T))=1$. In the notation of $\S 1$ we have $\alpha\left(\widetilde{\mathcal{P}}_{\varrho}\right)=\mathbb{N}$ for each $\varrho \in \widetilde{Q}$, while for each $k$ in $\mathbb{N}$ and $\varrho \in \widetilde{Q}$ we have $P_{k}(\varrho)=\widetilde{P}\left(i_{1}, \ldots, i_{k}\right)$. Thus for $\varrho \in \widetilde{Q}(T)$ we obtain

$$
\begin{aligned}
\liminf _{\beta \rightarrow \alpha\left(\widetilde{P}_{\varrho}\right)} \frac{\log \nu\left(P_{\beta}(\varrho)\right)}{\log \lambda \circ \pi\left(P_{\beta}(\varrho)\right)} & =\liminf _{k \rightarrow \infty} \frac{\log \nu\left(\widetilde{P}\left(i_{1}, \ldots, i_{k}\right)\right)}{\log \lambda\left(\phi_{i_{1}} \circ \ldots \circ \phi_{i_{k}}(Q)\right)} \\
& =\frac{h(T)}{\sum_{1 \leq i \leq n} a_{i}(T)},
\end{aligned}
$$


and so by Theorem 2.1 we have

$$
\operatorname{dim}_{\lambda \circ \pi}^{\mathcal{P}}(\widetilde{Q}(T)) \geq \frac{h(T)}{\sum_{1 \leq i \leq n} a_{i}(T)} .
$$

Since clearly $\pi(\widetilde{Q}(T))=E(T)$, Theorem 3.1 implies

$$
\operatorname{dim}_{\lambda}^{\mathcal{P}}(E(T)) \geq \frac{h(T)}{\sum_{1 \leq i \leq n} a_{i}(T)} .
$$

Now for each $M>0$ denote by $E(T, M)$ the set of $x$ in $E(T)$ such that $\left(i_{j}\right)_{j=1}^{\infty}$ in $W(x) \cap A(T)$ implies

$$
\left|\#\left(\left\{j \leq k: i_{j}=h\right\}\right)-k \tau_{h}\right| \leq M(k \log k)^{1 / 2},
$$

for all $h \in[1, n]$ and all $k$ in $\mathbb{N}$. Thus

$$
E(T)=\bigcup_{M>0} E(T, M) .
$$

Let $\mathcal{P}(T, M)$ be the subset of $\mathcal{P}$ consisting of the sets $\phi_{i_{1}} \circ \ldots \circ \phi_{i_{k}}(Q)$ where $i_{1}, \ldots, i_{k}$ is in the initial segment of some $\left(i_{j}\right)_{j=1}^{\infty}$ in $W(x) \cap A(T)$ for some $x$ in $E(T, M) \subset Q_{\mathcal{P}(T, M)}$. Moreover we claim that the hypotheses of Theorem 3.2 are satisfied with $X=Q, \mathcal{P}=\mathcal{P}(T, M), \eta=\lambda$ and

$$
\alpha=\frac{\sum_{1 \leq i \leq n} a_{i}(T)}{\min _{1 \leq i \leq n} a_{i}(T)} .
$$

In fact (N5) is obvious and (N6) is easily verified with $K=\min _{1 \leq j \leq t} \lambda\left(\phi_{j}(Q)\right)$. To check that $\left(\mathrm{N} 7_{\alpha}\right)$ holds observe that for each element $P=\phi_{i_{1}} \circ \ldots \circ \phi_{i_{k}}(Q)$ in $\mathcal{P}(T, M)$ we have

and so

$$
|\log | P||=\min _{1 \leq j \leq t} \sum_{1 \leq h \leq k}\left|\log a_{i}^{\left(i_{h}\right)}\right|
$$

$$
|\log | P|| \asymp \min _{1 \leq i \leq n} a_{i}(T) .
$$

Since

$$
|\log \lambda(P)| \asymp \sum_{1 \leq i \leq n} a_{i}(T),
$$

we see that $\left(\mathrm{N} 7_{\alpha}\right)$ holds as claimed. Now applying Theorem 3.2 we obtain

$$
\operatorname{dim} E(T) \geq n-\alpha+\alpha \operatorname{dim}_{\lambda}^{\mathcal{P}(T, M)}(E(T, M)) .
$$

Since $\mathcal{P}(T, M) \subset \mathcal{P}$ the definition of Billingsley dimension yields

$$
\operatorname{dim}_{\lambda}^{\mathcal{P}(T, M)} \geq \operatorname{dim}_{\lambda}^{\mathcal{P}} .
$$

Also by a standard argument ([Bi3], pp. 136-137) we have both

$$
\operatorname{dim} E(T)=\sup _{M} \operatorname{dim} E(T, M)
$$


and

$$
\operatorname{dim}_{\lambda}^{\mathcal{P}}(E(T))=\sup _{M} \operatorname{dim}_{\lambda}^{\mathcal{P}}(E(T, M)) .
$$

Thus letting $M \rightarrow \infty$ in (4.4) and using (4.2) and (4.3) we obtain

$$
\operatorname{dim} E \geq \operatorname{dim} E(T) \geq f(T),
$$

as required.

If condition (C) holds then for each $\alpha$ given by (4.3) we have $\alpha=n$ and so the preceding argument shows that $\operatorname{dim} E \geq n \operatorname{dim}_{\lambda}^{\mathcal{P}}(E)$ for all $E \subset Q$. The reverse inequality follows from the definition of Hausdorff and Billingsley dimensions so for $E \subset Q$ in the presence of condition (C) we have

$$
\operatorname{dim} E=n \operatorname{dim}_{\lambda}^{\mathcal{P}}(E) .
$$

5. Proof of Theorem 1.2. For each $h$ in $\Lambda$ and $T$ in $\mathcal{T}$ (not necessarily in $\left.\mathcal{T}_{\Phi}^{*}\right)$ we denote by $A_{h}^{*}(T)$ the set of sequences $\left(i_{k}\right)_{k=1}^{\infty}$ in $\Lambda^{\mathbb{N}}$ such that for each pair $(a, b)$ of integers with $a \geq 0, b \geq 1$ we have

$$
\left|\#\left(\left\{j \leq k: j \equiv a(\bmod b), i_{j}=h\right\}\right)-k \tau_{h} / b\right| \ll(k \log k)^{1 / 2} .
$$

We also define

$$
\begin{gathered}
E_{h}^{*}(T)=\left\{x \in E_{\Phi}(T): W(x) \cap A_{h}^{*}(T) \neq \emptyset\right\}, \\
A^{*}(T)=\bigcap_{1 \leq h \leq t} A_{h}^{*}(T), \quad E^{*}(T)=\bigcap_{1 \leq h \leq t} E_{h}^{*}(T) .
\end{gathered}
$$

By elementary probability arguments quoted from [Fel] we have

$$
\operatorname{dim}_{\lambda}^{\mathcal{P}}\left(E^{*}(T)\right)=\operatorname{dim}_{\lambda}^{\mathcal{P}}(E(T)),
$$

and as in the proof or Theorem 1.1 we obtain

$$
\operatorname{dim}_{\lambda}^{\mathcal{P}}\left(E^{*}(T)\right)=\frac{h(T)}{\sum_{1 \leq i \leq n} a_{i}(T)}=g(T),
$$

say. We can apply Theorem 3.2 with $\alpha$ given by (4.3) to obtain

$$
f(T) \leq \operatorname{dim} E^{*}(T) \leq n g(T) .
$$

If condition (C) holds, the remark following the proof of Theorem 1.1 implies that

$$
\operatorname{dim} E^{*}(T)=n \operatorname{dim}_{\lambda}^{\mathcal{P}}\left(E^{*}(T)\right)
$$

and also $\alpha=n$ implies $f(T)=n g(T)$ in the case where $T$ is in $\mathcal{T}_{\Phi}^{*}$. This means that

$$
\operatorname{dim} E^{*}\left(T_{0}\right)=f\left(T_{0}\right) .
$$

Now for non-negative integers $r, s$ with $0 \leq r \leq s$ we denote by $A^{*}(r, s ; T)$ the set of sequences $\left(i_{k}\right)_{k=1}^{\infty}$ in $A^{*}(T)$ such that for some $l \in \mathbb{Z}$ and all large 
$a \in \mathbb{N}$ satisfying $a \equiv l(\bmod s)$ we have $i_{a}=\ldots=i_{a+s-r}$. We also set

$$
E^{*}(r, s ; T)=\left\{x \in E_{\Phi}(Q): W(x) \cap A^{*}(r, s ; T) \neq \emptyset\right\} .
$$

We claim that

$$
\operatorname{dim} E^{*}\left(r, s ; T_{0}\right)=\frac{r}{s} \operatorname{dim} E^{*}\left(T_{0}\right)
$$

By the remark following the proof of Theorem 1.1, we have

$$
\operatorname{dim} E^{*}\left(T_{0}\right)=n \operatorname{dim}_{\lambda}^{\mathcal{P}}\left(E^{*}\left(T_{0}\right)\right),
$$

and also

$$
\operatorname{dim} E^{*}\left(r, s ; T_{0}\right)=n \operatorname{dim}_{\lambda}^{\mathcal{P}}\left(E^{*}\left(r, s ; T_{0}\right)\right),
$$

so we need to show that

$$
\operatorname{dim} E^{*}\left(r, s ; T_{0}\right)=\frac{r}{s} \operatorname{dim}_{\lambda}^{\mathcal{P}}\left(E^{*}\left(r, s ; T_{0}\right)\right)
$$

To see this we first split $E^{*}\left(r, s ; T_{0}\right)$ into finitely many pieces corresponding to the possible choices of $l(\bmod s)$, and then apply Theorem 2.2 with appropriate $\mu$ and $\nu$. For example in the case $l \equiv 0(\bmod s)$ we would take $\mu$ to be the product of discrete probability measures $p_{k}$ over $k \in \mathbb{N}$ where each $p_{k}$ is the distribution of the random variable $i_{k}$ given by the probability vector $T_{0}$, and we could take $\nu$ to be the product of measures $p_{k}^{r, s}$ where if $k$ is congruent to one of $1, \ldots, s-r(\bmod s)$ we put $p_{k}^{r, s}=\delta\left(i_{k}, i_{s[k / s]}\right)$ (where $\delta$ is Kronecker's delta and $[y]$ denotes the largest integer not greater than $y$ ), and if $k$ is congruent to one of $s-r+1, \ldots, s(\bmod s)$ we put $p_{k}^{r, s}=p_{k}$. This proves the claim that

$$
\operatorname{dim} E^{*}\left(r, s ; T_{0}\right)=\frac{r}{s} \operatorname{dim} E^{*}\left(T_{0}\right)
$$

It is easy to see that $E^{*}\left(r, s ; T_{0}\right)$ is self-affine with respect to $\Phi$, and for each $\delta$ in $[0,1]$ so is the set

$$
E^{*}\left(\delta ; T_{0}\right)=\bigcup_{0 \leq r / s \leq \delta} E^{*}\left(r, s ; T_{0}\right)
$$

Moreover we have

$$
\begin{aligned}
\operatorname{dim} E^{*}\left(\delta ; T_{0}\right) & =\sup _{0 \leq r / s \leq \delta} E^{*}\left(r, s ; T_{0}\right)=\sup _{0 \leq r / s \leq \delta} \frac{r}{s} \operatorname{dim} E^{*}\left(T_{0}\right) \\
& =\delta \operatorname{dim} E^{*}\left(T_{0}\right)=\delta f\left(T_{0}\right)
\end{aligned}
$$

and the theorem is proved.

6. Some remarks on the lower bound in Theorem 1.1. It is natural to conjecture that in the statement of Theorem 1.2 we can replace $\left[0, f\left(T_{0}\right)\right]$ by $[0, M]$ where $M$ is the maximum of $\operatorname{dim} E_{\Phi}(S)$ as $S$ ranges over the bounded subsets of $\mathbb{R}^{n}$. How close does Theorem 1.2 come to this conjecture? This is the same as asking: how good is the lower bound in 
Theorem 1.1? To assess the quality of this lower bound we consider the following special case. From now on in this section we confine attention to $\mathbb{R}^{2}$ and for convenience of computation we replace the unit square $Q$ by a closed square $S$ of side $w$ with sides parallel to the coordinate axes. The sets $\phi_{1}(S), \ldots, \phi_{r}(S)$ will be quasi-disjoint "dominoes"; more precisely the sets will be horizontal dominoes of the form $[x, x+2] \times[y, y+1]$ and the sets $\phi_{r+1}(S), \ldots, \phi_{t}(S)$ will be vertical dominoes of the form $[x, x+1] \times[y, y+2]$. We write $s=t-r$ and may suppose without loss of generality that $r \leq s$ (otherwise interchange $r$ with $s$ and $x$ with $y$ ). We need not exclude the case $r=0$ though this case can in fact be treated without recourse to the methods of this paper. In the statement of the following theorem and from now on all logarithms are to base two.

Theorem 6.1. We have

$$
\max _{0 \leq \tau \leq 1 / 2} f(\tau) \leq \operatorname{dim} E_{\Phi}(S) \leq \min \left(\gamma_{1}, \gamma_{2}\right),
$$

where we write

$$
f(\tau)=\frac{2 \tau-1+\tau \log r+(1-\tau) \log s+H(\tau)}{\tau+\log w-1} ;
$$

here $H$ is the binary entropy function $H(\tau)=-\tau \log \tau-(1-\tau) \log (1-\tau)$ and $\gamma_{1}, \gamma_{2}$ are the least non-negative solutions of

$$
r+2^{\gamma_{1}} s=w^{\gamma_{1}}, \quad r / 2+2^{1-\gamma_{2}} s=(w / 2)^{\gamma_{2}}
$$

respectively.

Proof. We prove first the right hand inequality, which does not depend on Theorem 4.1 although it will be convenient to make use of the net $\mathcal{P}=$ $\bigcup_{n \geq 0} \mathcal{P}_{n}$, where we set $\mathcal{P}_{0}$ to be $S$ and for all $n \geq 1$ we define $\mathcal{P}_{n}$ to be the class of sets $\phi_{j}(P)$ with $1 \leq j \leq t$ and $P \in \mathcal{P}_{n-1}$ (one verifies without difficulty that $\mathcal{P}$ is indeed a net over $S$ ). We partition $\mathcal{P}_{n}$ into sets $\mathcal{P}_{n, m}$ for $0 \leq m \leq n$ where each element of $\mathcal{P}_{n, m}$ has the form $\phi_{i_{1}} \circ \ldots \circ \phi_{i_{n}}(S)$ with exactly $m$ of the indices $i_{1}, \ldots, i_{n}$ lying in $[1, r]$. Thus $\mathcal{P}_{n, m}$ consists of $\left(\begin{array}{l}n \\ m\end{array}\right) r^{m} s^{n-m}$ sets each having diameter at most $2^{n-m} w^{-n}$ if $m \leq n / 2$ and at most $2^{m} w^{-n}$ if $m \geq n / 2$.

To prove $\operatorname{dim} E \leq \gamma_{1}$, note that $\operatorname{dim} E$ is bounded above by the infimum of the $\gamma$ such that both

$$
\limsup _{n \rightarrow \infty} \sum_{m \leq n / 2}\left(\begin{array}{c}
n \\
m
\end{array}\right) r^{m} s^{n-m}\left(\frac{2^{n-m}}{w^{n}}\right)^{\gamma}<\infty
$$

and

$$
\limsup _{n \rightarrow \infty} \sum_{m \geq n / 2}\left(\begin{array}{c}
n \\
m
\end{array}\right) r^{m} s^{n-m}\left(\frac{2^{m}}{w^{n}}\right)^{\gamma}<\infty .
$$


Using the assumption that $r \leq s$ we see that (6.1) implies (6.2) and that in turn (6.1) is a consequence of

$$
r+2^{\gamma} s \leq w^{\gamma}
$$

(In fact (6.3) and (6.1) are equivalent for $r \leq s$ but we make no use of this fact.) We have proved that $\operatorname{dim} E \leq \gamma_{1}$.

To prove $\operatorname{dim} E \leq \gamma_{2}$, note that for $m \leq n / 2$ each element in $\mathcal{P}_{n, m}$ can be covered by $2^{n-2 m}$ squares each of diameter $2^{n-m} w^{-n}$. As in the proof that $\operatorname{dim} E \leq \gamma_{1}$ we see that $\operatorname{dim} E$ does not exceed the least $\gamma$ such that both

$$
\limsup _{n \rightarrow \infty} \sum_{m \leq n / 2}\left(\begin{array}{c}
n \\
m
\end{array}\right) r^{m} s^{n-m} 2^{n-2 m}\left(\frac{2^{m}}{w^{n}}\right)^{\gamma}<\infty
$$

and

$$
\limsup _{n \rightarrow \infty} \sum_{m \geq n / 2}\left(\begin{array}{c}
n \\
m
\end{array}\right) r^{m} s^{n-m} 2^{2 m-n}\left(\frac{2^{n-m}}{w^{n}}\right)^{\gamma}<\infty .
$$

Now (6.4) and (6.5) are consequences of (indeed for $r \leq s$ are equivalent to)

$$
\begin{aligned}
& r / 2+2^{1-\gamma} s \leq(w / 2)^{\gamma}, \\
& 2^{1-\gamma} r+s / 2 \leq(w / 2)^{\gamma},
\end{aligned}
$$

respectively, and we see that for $r \leq s,(6.6)$ implies (6.7), so $\operatorname{dim} E \leq \gamma_{2}$ as required.

We now prove the left hand inequality. In order to apply Theorems 3.1, 3.2 and Billingsley's comparison principle, we introduce the following notions. Note that each $x \in E$ has the form $\bigcap_{k \geq 1} \phi_{i_{1}} \circ \ldots \circ \phi_{i_{k}}(S)$ for some $\omega(x)=\left(i_{k}\right)_{k \geq 1}$ in $\{1, \ldots, t\}^{\mathbb{N}}$. Similarly each $\varrho \in \widetilde{E}$ has the form $\left\{\phi_{i_{1}} \circ \ldots \circ\right.$ $\left.\phi_{i_{k}}(S): k \in \mathbb{N}\right\}$ for some $\widetilde{\omega}(\varrho)=\left(i_{k}\right)_{k \geq 1}$ in $\{1, \ldots, t\}^{\mathbb{N}}$. Thus $\widetilde{\omega}(\varrho)=\omega(x)$ implies $\pi(\varrho)=x$. We denote by $\widetilde{P}\left(i_{1}, \ldots, i_{k}\right)$ the set of $\varrho \in \widetilde{E}$ such that the first $k$ terms of $\widetilde{\omega}(\varrho)$ are $i_{1}, \ldots, i_{k}$. Every element of $\widetilde{\mathcal{P}}$ is of this form. We have made the convention that $\widetilde{E}=\widetilde{P}(\phi)$. We also have $\pi\left(\widetilde{P}\left(i_{1}, \ldots, i_{k}\right)\right)=$ $\phi_{i_{1}} \circ \ldots \circ \phi_{i_{k}}(S)$.

Now for $\tau \in[0,1]$ set

$$
A(\tau)=\left\{\omega \in\{1, \ldots, t\}^{\mathbb{N}}:\left|\#\left\{j \leq k: 1 \leq i_{j} \leq r\right\}-k \tau\right| \ll k^{1 / 2}\right\}
$$

and set

$$
E(\tau)=\{x \in E: \omega(x) \in A(\tau)\}, \quad \widetilde{E}(\tau)=\{\varrho \in \widetilde{E}: \omega(\varrho) \in A(\tau)\} .
$$

Since

$$
\sup _{\tau \in[0,1]} \operatorname{dim} E(\tau) \leq \operatorname{dim} E,
$$

we now seek a lower bound for $\operatorname{dim} \widetilde{E}(\tau)$ (as a function of $\tau$ ). We shall now proceed to estimating $\operatorname{dim}_{\lambda \circ \pi}^{\widetilde{\mathcal{P}}}(\widetilde{E}(\tau))$ using the comparison principle, where $\lambda$ 
denotes two dimensional Lebesgue measure. Define $\nu: \widetilde{\mathcal{P}} \rightarrow[0,1]$ recursively by setting $\nu(\widetilde{E})=1$ and for $\left(i_{1}, \ldots, i_{k}\right)$ in $\{1, \ldots, t\}^{\mathbb{N}}$, setting

$$
\nu\left(\widetilde{P}\left(i_{1}, \ldots, i_{k}\right)\right)= \begin{cases}\frac{\tau}{r} \nu\left(\widetilde{P}\left(i_{1}, \ldots, i_{k-1}\right)\right) & \text { if } i_{k} \leq r, \\ \frac{1-\tau}{s} \nu\left(\widetilde{P}\left(i_{1}, \ldots, i_{k-1}\right)\right) & \text { if } i_{k}>r .\end{cases}
$$

One checks that $\nu$ is finitely additive and then a special case $[\mathrm{Pa}]$ of Kolmogorov's consistency theorem shows that $\nu$ can be extended to a Borel probability measure (also denoted by $\nu$ ) on $\widetilde{E}$. It is easy to verify that $\nu$ is non-atomic. By the strong law of large numbers, $\nu(\widetilde{E}(\tau))=1$ and this easily implies that

$$
\operatorname{dim}_{\nu}^{\widetilde{\mathcal{P}}}(\widetilde{E}(\tau))=1 .
$$

In the notation of $\S 2$ we have $\alpha\left(\widetilde{\mathcal{P}}_{\varrho}\right)=\mathbb{N}$ for each $\varrho \in \widetilde{E}$, and for each $k$ in $\mathbb{N}$ and $\varrho \in \widetilde{E}$ we have $P_{k}(\varrho)=\widetilde{P}\left(i_{1}, \ldots, i_{k}\right)$ where $\widetilde{\omega}(\varrho)=\left(i_{k}\right)_{k \geq 1}$. Thus for $\varrho \in \widetilde{E}(\tau)$ we have

$$
\begin{aligned}
\liminf _{\beta \rightarrow \alpha\left(\widetilde{\mathcal{P}}_{\varrho}\right)} \frac{\log \nu\left(P_{\beta}(\varrho)\right)}{\log \lambda \circ \pi\left(P_{\beta}(\varrho)\right)} & =\liminf _{k \rightarrow \infty} \frac{\log \nu\left(\widetilde{P}\left(i_{1}, \ldots, i_{k}\right)\right)}{\log \lambda\left(\phi_{i_{1}} \circ \ldots \circ \phi_{i_{k}}(S)\right)} \\
& =\frac{\tau \log \left(\frac{\tau}{r}\right)+(1-\tau) \log \left(\frac{1-\tau}{s}\right)}{\log \left(\frac{2}{w^{2}}\right)}
\end{aligned}
$$

and so by Theorem 2.1 we have

$$
\begin{aligned}
\operatorname{dim}_{\lambda \circ \pi}^{\widetilde{\mathcal{P}}}(\widetilde{E}(\tau)) & \geq \frac{\tau \log \left(\frac{\tau}{r}\right)+(1-\tau) \log \left(\frac{1-\tau}{s}\right)}{\log \left(\frac{2}{w^{2}}\right)} \\
& =\frac{\tau \log r+(1-\tau) \log s+H(\tau)}{2 \log w-1} .
\end{aligned}
$$

Now for each $M>0$ denote by $E(\tau, M)$ the set of $x$ in $E(\tau)$ such that

$$
\left|\#\left(\left\{j \leq k: 1 \leq i_{j} \leq r\right\}\right)-\tau k\right| \leq M k^{1 / 2}
$$

for all $k \in \mathbb{N}$, where $\omega(x)=\left(i_{k}\right)_{k \geq 1}$, so that

$$
E(\tau)=\bigcup_{M>0} E(\tau, M)
$$

Let $\mathcal{P}(\tau, M)$ be the subset of $\mathcal{P}$ consisting of the sets $\phi_{i_{1}} \circ \ldots \circ \phi_{i_{k}}(S)$ where $i_{1}, \ldots, i_{k}$ is an initial segment of $\omega(x)$ for some $x$ in $E(\tau, M)$. Then one easily verifies that $\mathcal{P}(\tau, M)$ is a net over $S$ and $E(\tau, M) \subset S_{\mathcal{P}(\tau, M)}$. Moreover we claim that for $\tau \leq 1 / 2$ the hypotheses of Theorem 3.2 are satisfied with $S=X, \mathcal{P}=\mathcal{P}(\tau, M), \eta=\lambda$ and

$$
\alpha=\frac{2 \log w-1}{\tau+\log w-1} .
$$


In fact (N5) is obvious and (N6) is easily verified with $K=2 / w^{2}$. To check that $\left(\mathrm{N} 7_{\alpha}\right)$ holds we observe that for an element $P=\phi_{i_{1}} \circ \ldots \circ \phi_{i_{k}}(S)$ in $\mathcal{P}(\tau, M)$ we have

$$
|P|=\max \left(2^{k_{1}} w^{-k}, 2^{k_{2}} w^{-k}\right)
$$

where $k_{1}$ and $k_{2}$ are the cardinalities of the sets $\left\{j \leq k: i_{j} \leq r\right\}$ and $\left\{j \leq k: i_{j}>r\right\}$ respectively. Thus $\tau \leq 1 / 2$ implies

$$
|P| \ll 2^{(1-\tau) k} w^{-k},
$$

and since $\lambda(P)=\left(2 / w^{2}\right)^{k}$ we see that $\left(\mathrm{N} 7_{\alpha}\right)$ holds as required. Now applying Theorem 3.2 we obtain

$$
\operatorname{dim} E(\tau, M) \geq 2-\alpha+\alpha \operatorname{dim}_{\lambda}^{\mathcal{P}(\tau, M)}(E(\tau, M)) .
$$

Since $\mathcal{P}(\tau, M) \subset \mathcal{P}$ we have by the definition of Billingsley dimension

$$
\operatorname{dim}_{\lambda}^{\mathcal{P}(\tau, M)} \geq \operatorname{dim}_{\lambda}^{\mathcal{P}} .
$$

A straightforward computation (cf. [Bi3], pp. 136-137) shows that the Billingsley dimension, like Hausdorff dimension, is countably subadditive and so we have both

$$
\operatorname{dim} E(\tau)=\sup _{M} \operatorname{dim} E(\tau, M),
$$

and

$$
\operatorname{dim}_{\lambda}^{\mathcal{P}}(E(\tau))=\sup _{M} \operatorname{dim}_{\lambda}^{\mathcal{P}}(E(\tau, M)) .
$$

Thus letting $M \rightarrow \infty$ in (6.11) and using (6.8) and (6.9) we find that

$$
\operatorname{dim} E \geq \operatorname{dim} E(\tau) \geq f(\tau),
$$

for each $\tau \in[0,1 / 2]$ and so Theorem 6.1 is proved.

For some values of $r, s$ and $w$ the lower bound in Theorem 6.1 can be much simplified. If $r=0$ then $f(\tau)=\infty$ except at $\tau=0$ so we have

$$
\max _{0 \leq \tau \leq 1 / 2} f(\tau)=f(0)=\frac{\log s-1}{\log w-1} .
$$

If $r>0$ we will show that $f^{\prime}(\tau)$ is decreasing on $[0,1 / 2]$, in fact even on $[0,1]$ we have

$$
f^{\prime}(\tau)=\frac{(2+\log r-\log s-\log \tau+\log (1-\tau)) \log w-1+\log \tau-\log r)}{(1-\tau-\log w)^{2}} .
$$

We must have $\log w \geq 1$ (otherwise $r=s=0$ ) so the denominator is increasing in $(0,1)$, and the numerator is increasing because its derivative is

$$
\frac{1-\tau-\log w}{\tau(1-\tau)}
$$


and this is negative for $\tau \in(0,1)$. Thus $f^{\prime}$ is decreasing as claimed. Therefore $f$ has just one maximum in $[0,1 / 2]$ and this is attained at $1 / 2$ or in $[0,1 / 2)$ according to whether $f^{\prime}(1 / 2) \geq 0$ or $f^{\prime}(1 / 2)<0$, that is, according to whether

$$
(4 r)^{\log w-1} \geq s^{\log w} \quad \text { or } \quad(4 r)^{\log w-1}<s^{\log w} .
$$

Thus if $(4 r)^{\log w-1} \geq s^{\log w}$ we have

$$
\max _{0 \leq \tau \leq 1 / 2} f(\tau)=f\left(\frac{1}{2}\right)=\frac{2+\log r+\log s}{2 \log w-1} .
$$

If on the other hand $(4 r)^{\log w-1}<s^{\log w}$ then the maximum is attained at the unique zero of $f^{\prime}$ in $[0,1 / 2)$, that is, the unique solution of

$$
\frac{\tau^{\log w-1}}{(1-\tau)^{\log w}}=2^{2 \log w-1} \frac{r^{\log w-1}}{s^{\log w}} .
$$

There seems to be no simple formula for the $\max f^{\prime}(\tau)$ in this case, unless (6.13) can be explicitly solved. If however $w$ is a power of two then (6.13) becomes a polynomial, for example if $S$ is a standard chessboard we have $w=8$ and (6.13) becomes

$$
8 r^{2}(\tau-1)^{3}+s^{3} \tau^{2}=0
$$

The reader may wonder why we have apparently ignored the possibility of obtaining a better lower bound in Theorem 6.1 by considering values of $\tau$ in $(1 / 2,1]$ as well as in $[0,1 / 2]$. The reason is as follows. If $\tau>1 / 2$ then (6.10) no longer yields $|P| \ll 2^{(1-\tau) k} w^{-k}$ but only $|P| \leq 2^{\tau k} w^{-k}$; to obtain the condition $\left(\mathrm{N} 7_{\alpha}\right)$ we must then take

$$
\alpha=\frac{2 \log w-1}{\log w-\tau}
$$

instead of (6.9) and hence $f(\tau)$ must be replaced by

$$
g(\tau)=\frac{1-2 \tau+\tau \log r+(1-\tau) \log s+H \tau}{\log w-\tau} .
$$

Now an argument similar to that leading to (6.12) shows that $g$ is increasing on a subinterval $[1 / 2,1]$ if and only if $(4 s)^{\log w-1}<r^{\log w}$. We claim that this cannot happen, given our assumptions that $r \leq s$ and that $\phi_{j}(S)$ are quasi-disjoint. In fact $(4 s)^{\log w-1}<r^{\log w}$ together with $r \leq s$ would imply that $s>w / 4$, and the quasi-disjointness of the $\phi_{j}(S)$ implies $r+s \leq w^{2} / 2$, so $r<w / 4$. But then, as is easily checked,

$$
(4 s / r)^{\log w}>4 s,
$$

contradicting $(4 s)^{\log w-1}<s^{\log w}$. This shows that $g$ is decreasing on $[1 / 2,1]$. Since $g(1 / 2)=f(1 / 2)$ we have lost nothing ignoring the values of $\tau \in$ $(1 / 2,1]$. 
A natural question is how sharp the bounds given in Theorem 6.1 are. The following argument shows that the upper bound is usually exact, in a sense that we shall make precise. Observe that for given integers $r_{0}$ and $s_{0}$, if $w_{0}$ is the least positive number for which there exists a $\Phi$ with $(r, s, w)=$ $\left(r_{0}, s_{0}, w_{0}\right)$, then for any $w_{1}>w_{0}$ there exists a $\Phi$ with $(r, s, w)=\left(r_{0}, s_{0}, w_{1}\right)$, with the images $\Phi(S)$ lying in disjoint open subsets of $S$. Now fix $(r, s, w)=$ $\left(r_{0}, s_{0}, w_{1}\right)$ with $w_{1}>w_{0}$. Following [Fa1], for each sequence $i_{1}, \ldots, i_{n}$ in $\{1, \ldots, t-1\}^{n}$ with exactly $m$ components in $[1, r]$ and for some $\gamma$ in $[0,2]$ set

$$
F^{\gamma}\left(\phi_{i_{1}} \circ \ldots \circ \phi_{i_{h}}\right)= \begin{cases}\left(2^{n-m} w^{-n}\right)^{\gamma} & \text { if } \gamma \leq 1, m \leq n / 2, \\ \left(2^{m} w^{-n}\right)^{\gamma} & \text { if } \gamma \leq 1, m \geq n / 2, \\ \left(2^{n-m} w^{-n}\right)\left(2^{m} w^{-n}\right)^{\gamma-1} & \text { if } \gamma \geq 1, m \leq n / 2, \\ \left(2^{m} w^{n}\right)\left(2^{n-m} w^{-n}\right)^{\gamma-1} & \text { if } \gamma \geq 1, m \geq n / 2,\end{cases}
$$

A special case of Theorem 6.3 in [Fa1] now asserts that if $w_{1}>6$ then there exists a $\Phi$ with $(r, s, w)=\left(r_{0}, s_{0}, w_{1}\right)$ such that

$$
\operatorname{dim} E_{\Phi}(S)=\inf \left\{\gamma: \sum_{n \in \mathbb{N}} \sum_{\left(i_{1}, \ldots, i_{n}\right) \in\{1, \ldots, t\}^{n}} F^{\gamma}\left(\phi_{i_{1}} \circ \ldots \circ \phi_{i_{n}}\right)<\infty\right\} .
$$

Substituting in the definition of $F^{\gamma}$ and comparing (6.1), (6.2), (6.4) and (6.5) we see that the right hand side of (6.14) is equal to $\min \left(\gamma_{1}, \gamma_{2}\right)$ and this establishes our assertion about the upper bound. It seems reasonable that the upper bound is always exact (i.e. we need not assume that $w_{1}>w_{0}$ or $w_{1}>6$ ) but we have no proof of this.

The lower bound is certainly not exact for arbitrary choices of $(r, s, w)$, in fact for $(r, s)=(0,1)$ and $w>2$ it is actually worse than the trivial bound $\operatorname{dim} E \geq 0$. For some choices of $(r, s, w)$ it is however easily seen to be exact. Suppose $r \leq s / 2$ and set $w=2 r+s$. A straightforward computation gives $f^{\prime}(2 r / w)=0$. Then since $2 r / w \leq 1 / 2$ we have

$$
\max _{\tau \in[0,1 / 2]} f(\tau)=f(2 r / w),
$$

and another computation gives $f(2 r / w)=1$. However if we take $S=$ $[0, w] \times[0, w]$ and define $\Phi$ by setting

$$
\Phi_{j}(S)= \begin{cases}{[2(j-1), 2 j] \times[0,1]} & \text { when } j \leq r, \\ {[j+r-1, j+r] \times[0,2]} & \text { when } j>r,\end{cases}
$$

then clearly $E_{\Phi}(S)=[0, w] \times\{0\}$. Thus $\operatorname{dim} E_{\Phi}(S)=1$ and the lower bound is attained. A similar argument using the fact that $f^{\prime}\left(2 r / w^{2}\right)=0$ shows that it is always attained when $r+s=w^{2} / 2$ provided of course that there is a $\Phi$ with the given $(r, s, w)$, which is the case precisely when $r, s$ and $w$ are all even integers. An interesting further question is whether there are any other values of $(r, s, w)$ for which the lower bound is attained and how we recognise them. 


\section{References}

[Ab1] A. G. Abercrombie, Subgroups and subrings of profinite rings, Math. Proc. Cambridge Philos. Soc. 116 (1994), 209-222.

[Ab2] - The Hausdorff dimension of some exceptional sets of p-adic integer matrices, J. Number Theory 53 (1995), 311-341.

[Ab3] —, Badly approximable p-adic integers, Proc. Indian Acad. Sci. 105 (1995), 123134.

[AN1] A. G. Abercrombie and R. Nair, An exceptional set in the ergodic theory of Markov maps of the interval, Proc. London Math. Soc. (3) 75 (1997), 221-240.

[AN2] - - - An exceptional set in the ergodic theory of expanding maps of manifolds, preprint.

[Be] A. F. Beardon, On the Hausdorff dimension of self-affine fractals, Proc. Cambridge Philos. Soc. 61 (1965), 679-694.

[Bed] T. Bedford, Ph.D. thesis, Univ. of Warwick, 1984.

[Bi1] P. Billingsley, Hausdorff dimension in probability theory, Illinois J. Math. 4 (1960), 187-209.

[Bi2] -, Hausdorff dimension in probability theory II, ibid. 5 (1965), 291-298.

[Bi3] - Ergodic Theory and Information, Wiley, New York, 1965.

[Caj] H. Cajar, Billingsley Dimension in Probability Spaces, Lecture Notes in Math. 892 Springer, 1981.

[Fa1] K. J. Falconer, The Hausdorff dimension of self-affine fractals, Math. Proc. Cambridge Philos. Soc. 103 (1988), 339-350.

[Fa2] - The Hausdorff dimension of self-affine fractals II, ibid. 111 (1992), 169-180.

[Fel] W. Feller, An Introduction to Probability Theory and its Applications I, Wiley, New York, 1968.

[HL] I. Hueter and S. P. Lalley, Falconer's formula for the Hausdorff dimension of a self-affine set in $\mathbb{R}^{2}$, Ergodic Theory Dynam. Systems 15 (1995), 77-97.

[KP] R. Kenyon and Y. Peres, Intersecting random translates of Cantor sets, Invent. Math. 104 (1991), 601-629.

[McM] C. McMullen, The Hausdorff dimension of general Sierpiński carpets, Nagoya Math J. 96 (1984), 1-9.

[Pa] K. R. Parthasarathy, Probability Measures on Metric Spaces, Academic Press, New York, 1967.

[SS] L. A. Steen and J. A. Seebach, Counterexamples in Topology, 2nd ed., Springer, New York, 1978.

Mathematical Sciences

University of Liverpool

P.O. Box 147

Liverpool L69 3BX, U.K.

E-mail: nair@liverpool.ac.uk

Received April 6, 1999

Revised version August 28, 2000 